\title{
ON THE ROUTE TO CHAOS FOR DOMAIN WALLS WITH A MODEL OF STRAY FIELD
}

\author{
A. Sukiennicki, R.A. Kosiński and J.J. Żebrowski \\ Institute of Physics, Warsaw University of Technology \\ Koszykowa 75, 00-662 Warszawa, Poland
}

(Received December 17, 1993)

\begin{abstract}
The route to chaos of domain wall in thin magnetic film, which is described by Slonczewski's equations of motion, is analyzed numerically. Hagedorn's model of surface stray field is applied. Ranges of periodic and chaotic wall motion as a function of constant in time, drive field are found. Comparison of results with those obtained for Hubert's model of the stray field is made.
\end{abstract}

PACS numbers: $05.45 .+\mathrm{b}, 75.10 . \mathrm{Hk}, 75.60 . \mathrm{Ch}$

\section{Introduction}

Domain walls in uniaxial magnetic materials exhibit an interesting chaotic dynamics. Some years ago it was shown theoretically that for drive fields larger than the critical field in an infinite material a structural transition occurs in the wall and a strange attractor appears in a properly chosen phase space [1]. On the other hand, recently it has been found for finite thickness samples that, if the magnetic stray fields coming from surfaces of the material are taken into account in the calculations, a transition to chaos is realized via intermittency [2]. For this purpose, an expression for the stray fields, proposed some time ago by Hubert [3], was used in [2].

In this note, a different expression for the stray fields is used, namely that given by Hagedorn [4]. It is found that, in this case, no intermittency is observed and the route to chaos is realized via a specific sequence of periodic states. It would be interesting to investigate the route to chaos experimentally in order to answer the question whether such a route in real bubble garnet films occurs through intermittency or not. 


\section{Equations of motion}

A twisted magnetic domain wall in the uniaxial magnetic bubble garnet film is described by a pair of nonlinear partial differential equations which can be derived from the Landau-Lifschitz equation with Gilbert's damping term included [5]:

$$
\begin{aligned}
& \frac{\dot{q}}{\Delta}=2 \pi M \gamma \sin 2 \psi-\frac{2 A \gamma}{M} \psi_{z z}+\frac{\gamma K}{M} \sin 2 \psi+\frac{\gamma \pi}{2} H_{\mathrm{sy}} \sin 2 \psi+\alpha \dot{\psi}, \\
& \dot{\psi}=\gamma H_{z}+\frac{2 \gamma A}{M \Delta} q_{z z}-\frac{\alpha}{\Delta} \dot{q},
\end{aligned}
$$

where the stray fields $H_{\text {sy }}$ from the surfaces of the film are included. Here, $A$ is the exchange constant, $\gamma$ is the gyromagnetic ratio, $4 \pi M$ is the saturation magnetization, $\alpha$ is the Gilbert damping constant, $\Delta=\sqrt{A / K}$ is the wall thickness parameter ( $K$ is the uniaxial perpendicular anisotropy constant), $H_{z}$ is the constant drive field applied to the film. In Eqs. (1), (2) $q(z, t)$ describes the local position of the wall and the azimuthal angle $\psi(z, t)$ describes the direction of the local magnetic moment of the wall with respect to $+O x$ axis. The coordinate $z$ is perpendicular to the film and parallel to the easy axis of the uniaxial anisotropy of the film. A dot over a symbol denotes the time derivative and the subscript $z z$ - the second derivative with respect to the $z$ coordinate.

The stray fields $H_{\text {sy }}$ from the surfaces of the film are calculated here according to the model proposed by Hagedorn [4] and equal

$$
\begin{aligned}
H_{\mathrm{sy}} & =-2 M\left\{\ln \frac{(h / 2+z)^{2}+\Delta^{2} / 4}{(h / 2-z)^{2}+\Delta^{2} / 4}+\frac{4}{\Delta}\left[(h / 2+z) \arctan \frac{\Delta}{(h+2 z)}\right.\right. \\
& \left.\left.-(h / 2-z) \arctan \frac{\Delta}{(h-2 z)}\right]\right\},
\end{aligned}
$$

where $h$ denotes the film thickness. This model takes into account the fact that the surface poles are modified by the finite wall width, while in the model proposed by IIubert [3], used in Ref. [2], a special wall width dependent transformation is applied to avoid singularities at the surfaces of the film. Both models are however very simplified. In any case both models due to their compact forms enable to analyze effectively the most important features of wall motion and have been used in the literature.

Equations of motion (1) and (2) were solved by means of a full implicit numerical scheme which is described in Ref. [6]. Force-free boundary conditions were applied [5]. The initial conditions were $q(z, t)=0, \psi(z, 0)=\psi_{\mathrm{s}}(z)$, where $\psi_{s}(z)$ is the static distribution for the twisted wall [5].

The material parameters were taken the same as in Ref. [2]. Namely, $A=$ $0.81 \times 10^{-7} \mathrm{erg} / \mathrm{cm}, 4 \pi M=140 \mathrm{G}, \gamma=1.75 \times 10^{7} \mathrm{~s}^{-1} \mathrm{Oe}^{-1}, \Delta=2.9 \times 10^{-6} \mathrm{~cm}$, $\alpha=0.156, h=1.4 \times 10^{-4} \mathrm{~cm}$.

\section{Method of analysis}

To analyze the type of motion of the wall, the trajectory $\tilde{q}(\tilde{\psi})$ of the mid-point of the wall was calculated at each time step of the integration procedure. The tilde denotes that the values of $\bar{q}$ and $\bar{\psi}$ averaged over the thickness 
of the film were subtracted from the instantaneous values of $q$ and $\psi$, respectively, because in this paper we are interested in the wall structure during the motion but not the overall translational motion of the wall. Only asymptotical trajectories are analyzed, i.e. such for which all transients are ceased.

\section{Results}

For drive fields $H_{z}$ very small, the point attractor was obtained and for intermediate values of $H_{z}$ the periodic attractor with a characteristic double-loop shape was found, similarly as in Ref. [2] (see Fig. 1 here and compare with Fig. 4 of Ref. [2]). For drives larger than a certain value of about $26.67 \mathrm{Oe}$ another periodic

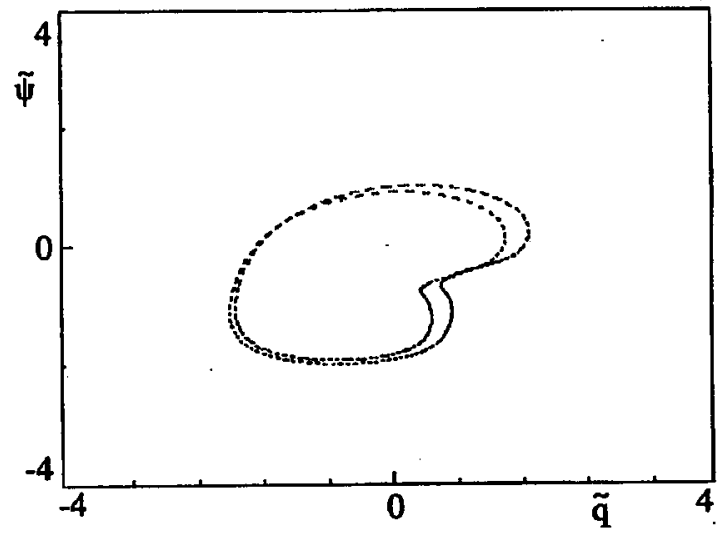

Fig. 1. Periodic attractor for $H_{z}=26.675 \mathrm{Oe}$.

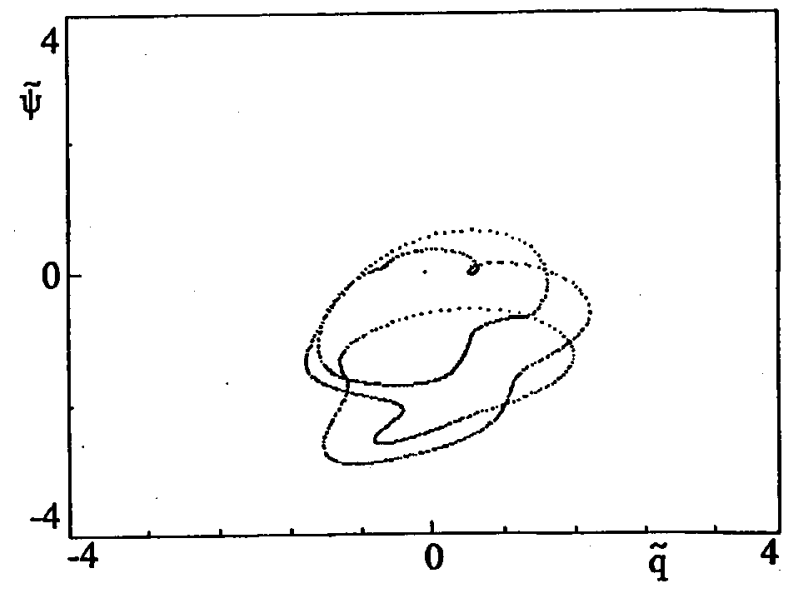

Fig. 2. Periodic attractor for $H_{z}=26.677$ Oe. 


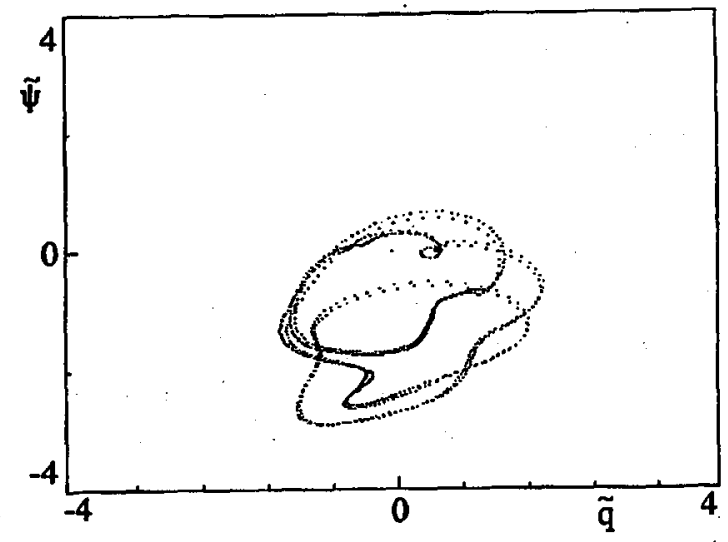

Fig. 3. Periodic attractor for $H_{z}=26.70 \mathrm{Oe}$.

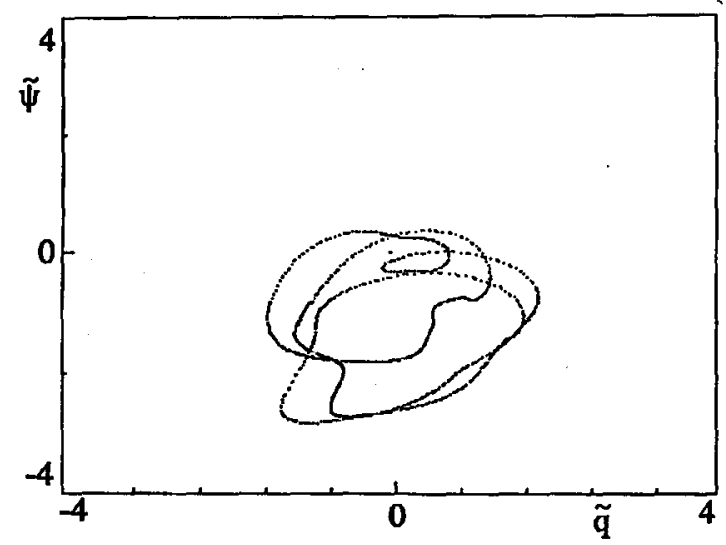

Fig. 4. Periodic attractor for $H_{z}=26.90$ Oe.

attractor appears (Fig. 2) and for $H_{z}=26.686$ Oe we find a similar attractor as the last one but with the period doubled (Fig. 3). This kind of attractor (with a small deformation) is found still at $H_{z}=26.80$ Oe but at 26.90 Oe all traces of doubled period disappear and the shape of the attractor is basically the same as at $26.68 \mathrm{Oe}$ (Fig. 4). Finally at drives higher than $H_{z}=26.92$ Oe the chaotic attractor is obtained (Fig. 5), with exception of some periodic windows (e.g. for $H_{z}=35.00 \mathrm{Oe}$ ), where the shape of the attractor is similar to that in Fig. 1 .

Comparing with the results obtained in Ref. [2], we find that the route to chaos depends strongly on the form of the stray fields coming from the surface magnetic poles. The use of Hagedorn's expression for the stray fields leads to a more complicated route to chaos than that found for Hubert's expression of [2]. Instead of the route via intermittency, the route to chaos is realized via a specific 


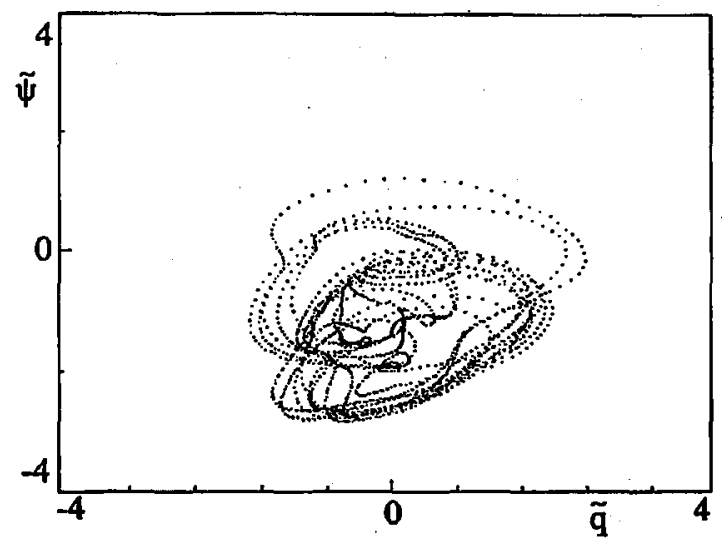

Fig. 5. Chaotic attractor for $H_{z}=26.95$ Oe.

sequence of periodic states and no traces of intermittency are found in the present case. Similarly as in [2], the route to chaos culminates in a collision between two attractors in phase space. This may be seen from the shape of the trajectories in Fig. 5 in which traces of both the periodic attractors of Fig. 1 and of Fig. 2 can be easily identified. Note, however, that here the competing attractors are both periodic while in Ref. [2] one of them was chaotic. The reason for such qualitative change in the dynamics between two models of the motion of the twisted wall seems to lie in the way the two kinds of surface stray field expressions treat the solitary wave (Bloch line) which appears in the structure of the wall as this wave reflects at the surfaces of the film. With Hagedorn's model of the stray field, a barrier of a monotonically increasing albeit finite height is encountered by the solitary wave as it approaches the surface of the film. In Hubert's model the barrier decreases sharply close to the surface thereby making the rotation of the magnetic moment at the surface of the film (necessary for the reflection of the Bloch line to occur) easier. As a result then, the two different models represent two significantly different types of boundary layer. It is interesting then that the type of a somewhat thin boundary layer influences the route to chaos of this, after all, a spatially extended system as a whole in such a qualitative manner.

It would be interesting to investigate the transition to chaos experimentally. Note at this point that the formula of IIagedorn has been shown to predict the critical field for the transition to chaotic solutions reasonably well [7] although the concept of chaos and so the route to it was not studied at that time. The formula of Hagedorn was also tested in [8] with good results against experimental data on the apparent width of the dynamic diffuse wall observed in [9] - but again the route to chaos was not studied then. On the other hand, the values of the peak velocity of the wall in the presence of the in-plane field, obtained numerically with the formula of Hubert, agree quite well [6] with the experimental data found in the rocking method experiment [10]. To conclude, it would seem interesting to investigate again domain wall dynamics in bubble garnet materials in the range 
of drive fields around the transition to dynamic Bloch line stacking - this time within the context of the concept of deterministic chaos - this could shed new light on the transition discussed here.

\section{Acknowledgment}

This work was supported by grant of the Committee for Scientific Research, No. 223219102.

\section{References}

[1] J.J. Żebrowski, A. Sukiennicki, Acta Phys. Pol. A 72, 299 (1987).

[2] A. Sukiennicki, R.A. Kosiński, J. Magn. Magn. Maler. 129, 213 (1994).

[3] A. Hubert, J. Appl. Phys. 46, 2276 (1975).

[4] F.B. Hagedorn, J. Appl. Phys. 45, 3129 (1974).

[5] A.P. Malozemoff, J.C. Slonczewski, Magnetic Domain Walls in Bubble Materials, American Press, New York 1979.

[6] R.A. Kosiński, J. Engemann, J. Magn. Magn. Mater. 50, 229 (1985).

[7] J.J. Żebrowski, J. Appl. Phys. 56, 249 (1984).

[8] R.A. Kosiński, J. Phys. C (France) 8, 632 (1988).

[9] K. Vural, F.B. Humphrey, J. Appl. Phys. 51, 549 (1980).

[10] R.A. Kosiniski, J. Heidmann, D. Krumbholz, J. Engemann, IEEE Trans. Magn. MAG-20, 1150 (1984). 